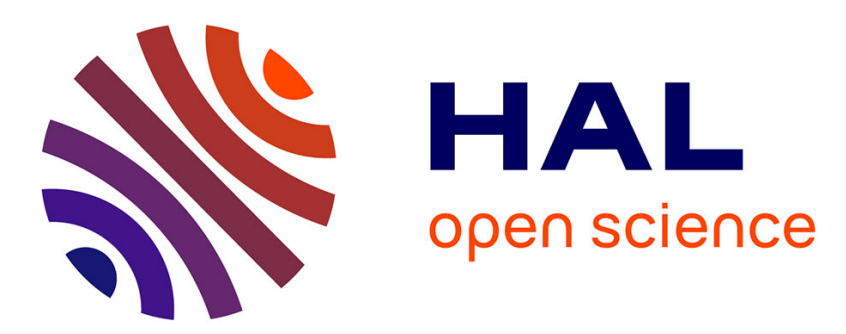

\title{
Integrability of invariant metrics on the diffeomorphism group of the circle
}

\author{
Adrian Constantin, Boris Kolev
}

\section{To cite this version:}

Adrian Constantin, Boris Kolev. Integrability of invariant metrics on the diffeomorphism group of the circle. Journal of Nonlinear Science, 2006, 16, pp.109-122. 10.1007/s00332-005-0707-4 . hal00095916

\section{HAL Id: hal-00095916 https://hal.science/hal-00095916}

Submitted on 26 Nov 2009

HAL is a multi-disciplinary open access archive for the deposit and dissemination of scientific research documents, whether they are published or not. The documents may come from teaching and research institutions in France or abroad, or from public or private research centers.
L'archive ouverte pluridisciplinaire HAL, est destinée au dépôt et à la diffusion de documents scientifiques de niveau recherche, publiés ou non, émanant des établissements d'enseignement et de recherche français ou étrangers, des laboratoires publics ou privés. 


\title{
INTEGRABILITY OF INVARIANT METRICS ON THE DIFFEOMORPHISM GROUP OF THE CIRCLE
}

\author{
ADRIAN CONSTANTIN AND BORIS KOLEV
}

\begin{abstract}
Each $H^{k}$ Sobolev inner product $(k \geq 0)$ defines a Hamiltonian vector field $X_{k}$ on the regular dual of the Lie algebra of the diffeomorphism group of the circle. We show that only $X_{0}$ and $X_{1}$ are bi-Hamiltonian relatively to a modified Lie-Poisson structure.
\end{abstract}

\section{INTRODUCTION}

Often motions of inertial mechanical systems are described in Lagrangian variables by paths on a configuration space $G$ that is a Lie group. The velocity phase space is the tangent bundle $T G$ and the kinetic energy

$$
\mathcal{K}=\frac{1}{2}\langle v, v\rangle
$$

for $v \in T G$. For example, in continuum mechanics the state of a system at time $t \geq 0$ can be specified by a diffeomorphism $x \mapsto \varphi(t, x)$ of the ambient space, giving the configuration of the particles with respect to their initial positions at time $t=0$. Here $x$ is a label identifying a particle, taken to be the position of the particle at time $t=0$ so that $\varphi(0, x)=x$. In this setting $G$ would be the group of diffeomorphisms. The material (Lagrangian) velocity field is given by $(t, x) \mapsto \varphi_{t}(t, x)$ while the spatial (Eulerian) velocity field is $u(t, y)=\varphi_{t}(t, x)$, where $y=\varphi(t, x)$, i.e. $u=\varphi_{t} \circ \varphi^{-1}$. Observe that for any fixed time-independent diffeomorphism $\eta$, the spatial velocity field $u=\varphi_{t} \circ \varphi^{-1}$ along the path $t \mapsto \varphi(t)$ remains unchanged if we replace this path by $t \mapsto \varphi(t) \circ \eta$. This right-invariance property suggests to extend the kinetic energy $\mathcal{K}$ by right translation to a right-invariant Lagrangian $\mathcal{K}: T G \rightarrow \mathbb{R}$, obtaining a Lagrangian system on $G$. The length of a path $\{\varphi(t)\}_{t \in[a, b]}$ in $G$ is defined as

$$
\mathfrak{l}(\varphi)=\int_{a}^{b}<\varphi_{t}, \varphi_{t}>^{1 / 2} d t
$$

The Least Action Principle holds if the equation of motion is the geodesic equation. The set Diff( $\left.\mathbb{S}^{1}\right)$ of all smooth orientation-preserving diffeomorphisms of the circle represents the configuration space for the spatially periodic motion of inertial one-dimensional mechanical systems. Diff $\left(\mathbb{S}^{1}\right)$ is an infinite dimensional Lie group, the group operation being composition [19] and its Lie algebra Vect $\left(\mathbb{S}^{1}\right)$ being the space of all smooth vector fields on $\mathbb{S}^{1}$

Date: July 2005.

2000 Mathematics Subject Classification. 35Q35, 35Q53, 37K10, 37K65.

Key words and phrases. Bi-Hamiltonian formalism, Diffeomorphisms group of the circle, Camassa-Holm equation.

This research was supported by the SFI-grant 04/BR/M0042. 
cf. [29]. On the regular (or $\left.L^{2}\right)$ dual Vect* $\left(\mathbb{S}^{1}\right)$ of the Lie algebra Vect $\left(\mathbb{S}^{1}\right)$ there are some affine canonical Lie-Poisson structures, called modified LiePoisson structures, which are all compatible. On the other hand, we can consider on the regular dual Vect* $\left(\mathbb{S}^{1}\right)$ a countable family $\left\{X_{k}\right\}_{k \geq 0}$ of Hamiltonian vector fields defined by Sobolev inner products. The importance of these inner products lies in that each gives rise via right translation to a geodesic flow on Diff $\left(\mathbb{S}^{1}\right)$, the Riemannian exponential map of which defines a local chart for every $k \geq 1 \mathrm{cf}$. [10] - a property which fails for the Lie group exponential map $[19,27]$ as well as for the Riemannian exponential map if $k=0$ [9]. In this paper we show that the Hamiltonian vector field $X_{k}$ is bi-Hamiltonian relatively to a modified Lie-Poisson structure if and only if $k \in\{0,1\}$.

\section{Preliminaries}

In this section, we review some fundamental aspects of finite dimensional smooth Poisson manifolds.

Definition 2.1. A symplectic manifold is a pair $(M, \omega)$, where $M$ is a manifold and $\omega$ is a closed nondegenerate 2-form on $M$, that is $d \omega=0$ and for each $m \in M, \omega_{m}: T_{m} M \times T_{m} M \rightarrow \mathbb{R}$ is a continuous bilinear skew-symmetric map such that the induced linear map $\tilde{\omega}_{v}: T_{m} M \rightarrow T_{m}^{*} M$ defined by $\tilde{\omega}_{v}(w)=\omega(v, w)$ is an isomorphism for all $v \in T_{m} M$.

Example 2.2. In the general study of variational problems, extensive use is made of the canonical symplectic structure on the cotangent bundle $T^{*} M$ (representing the phase space) of the manifold $M$ (representing the configuration space). This symplectic form is given in any local trivialization $(q, p) \in U \times \mathbb{R}^{n} \subset \mathbb{R}^{n} \times \mathbb{R}^{n}$ of $T^{*} M$ by

$$
\omega_{(q, p)}((Q, P),(\tilde{Q}, \tilde{P}))=\tilde{P} \cdot Q-P \cdot \tilde{Q}, \quad(Q, P),(\tilde{Q}, \tilde{P}) \in \mathbb{R}^{n} \times \mathbb{R}^{n} .
$$

Since a symplectic form $\omega$ is nondegenerate, it induces an isomorphism

$$
\text { b }: T M \rightarrow T^{*} M, \quad X \mapsto X^{b},
$$

defined via $X^{b}(Y)=\omega(X, Y)$. The symplectic gradient $X_{f}$ of a function $f$ is defined by the relation $X_{f}^{b}=-d f$. The inverse of the isomorphism $b$ defines a skew-symmetric bilinear form $W$ on the cotangent space of $M$. This bilinear form $W$ induces itself a bilinear mapping on $\mathrm{C}^{\infty}(M)$, the space of smooth functions $f: M \rightarrow \mathbb{R}$, given by

$$
\{f, g\}=W(d f, d g)=\omega\left(X_{f}, X_{g}\right), \quad f, g \in \mathrm{C}^{\infty}(M),
$$

and called the Poisson bracket of the functions $f$ and $g$.

Example 2.3. In Example 2.2, the Poisson bracket is given by

$$
\{f, g\}=\sum_{i=1}^{n}\left(\frac{\partial f}{\partial q_{i}} \frac{\partial g}{\partial p_{i}}-\frac{\partial f}{\partial p_{i}} \frac{\partial g}{\partial q_{i}}\right) .
$$

The observation that a bracket like (2.3) could be introduced on $\mathrm{C}^{\infty}(M)$ for a smooth manifold $M$, without the use of a symplectic form, leads to the general notion of a Poisson structure [26]. 
Definition 2.4. A Poisson structure on a $C^{\infty}$ manifold $M$ is a skewsymmetric bilinear mapping $(f, g) \mapsto\{f, g\}$ on the space $\mathrm{C}^{\infty}(M)$, which satisfies the Jacobi identity

$$
\{\{f, g\}, h\}+\{\{g, h\}, f\}+\{\{h, f\}, g\}=0,
$$

as well as the Leibnitz identity

$$
\{f, g h\}=\{f, g\} h+g\{f, h\} .
$$

When the Poisson structure is induced by a symplectic structure $\omega$, the Leibnitz identity is a direct consequence of (2.2), whereas the Jacobi identity (2.4) corresponds to the condition $d \omega=0$ satisfied by the symplectic form $\omega$. In the general case, the fact that the mapping $g \mapsto\{f, g\}$ satisfies (2.5) means that it is a derivation of $\mathrm{C}^{\infty}(M)$. Each derivation on $\mathrm{C}^{\infty}(M)$ for a $C^{\infty}$ manifold (even in the infinite dimensional case cf. [1]) corresponds to a smooth vector field, that is, to each $f \in \mathrm{C}^{\infty}(M)$ is associated a vector field $X_{f}: M \rightarrow T M$, called the Hamiltonian vector field of $f$, such that

$$
\{f, g\}=X_{f} \cdot g=d g \cdot X_{f},
$$

where $d g . X_{f}=L_{X_{f}} g$ is the Lie derivative of $g$ along $X_{f}$. Conversely, a vector field $X: M \rightarrow T M$ on a Poisson manifold $M$ is said to be Hamiltonian if there exists a function $f$ such that $X=X_{f}$.

Recall [29] that for a smooth vector field $X: M \rightarrow T M$, the Lie derivative operator $L_{X}: \mathrm{C}^{\infty}(M) \rightarrow \mathrm{C}^{\infty}(M)$ acts on smooth functions $g: M \rightarrow \mathbb{R}$ with differentials $d g: M \rightarrow T^{*} M$ by $\left(L_{X} g\right)(m)=d g(m) \cdot X(m)$ for $m \in M$. The space $\operatorname{Vect}(M)$ of smooth vector fields on $M$ and the space of operators $\left\{L_{X}: X \in \operatorname{Vect}(M)\right\}$ are isomorphic as real vector spaces, the linear isomorphism between them being $X \mapsto L_{X}$ [1]. Therefore the elements of $\operatorname{Vect}(M)$ can be regarded as operators on $\mathrm{C}^{\infty}(M)$ via $X \cdot f=L_{X} f$, forming a Lie algebra if endowed with the bracket $[X, Y]=L_{X} \circ L_{Y}-L_{Y} \circ L_{X}$. Notice that (2.4) yields

$$
\left[X_{f}, X_{g}\right]=X_{\{f, g\}} \cdot
$$

From (2.7) it follows (see [29]) that $g \in \mathrm{C}^{\infty}(M)$ is a constant of motion for $X_{f}$ if and only if $\{f, g\}=0$.

Jost [21] pointed out that, just like a derivation on $\mathrm{C}^{\infty}(M)$ corresponds to a vector field, a bilinear bracket $\{f, g\}$ satisfying the Leibnitz rule (2.5) corresponds to a skew-symmetric bilinear form on $T M$. That is, there exists a $C^{\infty}$ tensor field $W \in \Gamma\left(\bigwedge^{2} T M\right)$, called the Poisson bivector of $(M,\{\cdot, \cdot\})$, such that

$$
\{f, g\}=W(d f, d g) .
$$

Using the unique local extension of the Lie bracket of vector fields to skewsymmetric multivector fields, called the Schouten-Nijenhuis bracket [30], the condition (2.4) becomes

$$
[W, W]=0 .
$$

Conversely, any $W \in \Gamma\left(\bigwedge^{2} T M\right)$ that satisfies (2.8) induces a Poisson structure on $M$ via (2.2). The only condition that must be satisfied by $W$ is (2.8) since (2.5) holds automatically. A Poisson structure on $M$ is therefore 
equivalent to a bivector $W$ that satisfies (2.8). This induces a homomorphism

$$
\#: T^{*} M \rightarrow T M, \quad \alpha \mapsto \alpha^{\#},
$$

such that $\beta\left(\alpha^{\#}\right)=W(\beta, \alpha)$ for every $\beta \in T^{*} M$. Notice that for $f \in \mathrm{C}^{\infty}(M)$ we have $(d f)^{\#}=X_{f}$. If the homomorphism (2.9) is an isomorphism we call the Poisson structure nondegenerate. A nondegenerate Poisson structure on $M$ is equivalent to a symplectic structure where the symplectic form $\omega$ is just $\# W$, the closedness condition corresponding to the Jacobi identity [30].

Remark 2.5. The notion of a Poisson manifold is more general than that of a symplectic manifold. For example, in the symplectic case the Poisson bracket satisfies the additional property that $\{f, g\}=0$ for all $g \in \mathrm{C}^{\infty}(M)$ only if $f \in \mathrm{C}^{\infty}(M)$ is constant, whereas for Poisson manifolds such nonconstant functions $f$ might exist, in which case they are called Casimir functions. To highlight this, notice that by Darboux' theorem [29] a finite dimensional symplectic manifold $M$ has to be even dimensional and locally there are coordinates $\left\{q_{1}, \ldots, q_{n}, p_{1}, \ldots, p_{n}\right\}$ such that $\{f, g\}$ is given by $(2.3)$. On the other hand, on $M=\mathbb{R}^{2 n+1}$ with coordinates $\left\{q_{1}, \ldots, q_{n}, p_{1}, \ldots, p_{n}, \zeta\right\}$ we determine a Poisson structure defining the Poisson bracket of $f, g \in$ $C^{\infty}\left(\mathbb{R}^{2 n+1}\right)$ by the same formula (2.3). Notice that any $f \in C^{\infty}\left(\mathbb{R}^{2 n+1}\right)$ which depends only on $\zeta$ is a Casimir function.

Two Poisson bivectors $W_{1}$ and $W_{2}$ define compatible Poisson structures if

$$
\left[W_{1}, W_{2}\right]=0 .
$$

This is equivalent to say that for any $\lambda, \mu \in \mathbb{R}$,

$$
\{f, g\}_{\lambda, \mu}=\lambda\{f, g\}_{1}+\mu\{f, g\}_{2}
$$

is also a Poisson bracket. On a manifold $M$ equipped with two compatible Poisson structures, a vector field $X$ is said to be (formally) integrable or bi-Hamiltonian if it is Hamiltonian for both structures.

On a symplectic manifold $(M, \omega)$, a necessary condition for a vector field $X$ to be Hamiltonian is that $L_{X} \omega=0$ [29]. A similar criterion exists for a Poisson manifold $(M, W)$. It is instructive for later considerations to present a short proof of this known result.

Proposition 2.6. On a Poisson manifold $(M, W)$ a necessary condition for a vector field $X$ to be Hamiltonian is

$$
L_{X} W=0 .
$$

Proof. If $X$ is Hamiltonian, there is a function $h \in \mathrm{C}^{\infty}(M)$ such that $X=$ $X_{h}$. Let $f$ and $g$ be arbitrary smooth functions on $M$. We have

$$
L_{X} W(d f, d g)=L_{X}(W(d f, d g))-W\left(L_{X} d f, d g\right)-W\left(d f, L_{X} d g\right) .
$$

But $L_{X_{h}} f=\{h, f\}$ and $L_{X_{h}} d f=d L_{X_{h}} f=d\{h, f\}$. Therefore

$$
\begin{aligned}
L_{X} W(d f, d g) & =L_{X}\{f, g\}-W(d\{h, f\}, d g)-W(d f, d\{h, g\}) \\
& =\{h,\{f, g\}\}-\{\{h, f\}, g\}-\{f,\{h, g\}\} .
\end{aligned}
$$

This last expression equals zero because of the Jacobi identity. 
The fundamental example of a non-symplectic Poisson structure is the Lie-Poisson structure on the dual $\mathfrak{g}^{*}$ of a Lie algebra $\mathfrak{g}$.

Definition 2.7. On the dual space $\mathfrak{g}^{*}$ of a Lie algebra $\mathfrak{g}$ of a Lie group $G$, there is a Poisson structure defined by

$$
\{f, g\}(m)=m\left(\left[d_{m} f, d_{m} g\right]\right)
$$

for $m \in \mathfrak{g}^{*}$ and $f, g \in C^{\infty}\left(\mathfrak{g}^{*}\right)$, called the canonical Lie-Poisson structure ${ }^{1}$.

Remark 2.8. The canonical Lie-Poisson structure has the remarkable property to be linear. A Poisson bracket on a vector space is said to be linear if the bracket of two linear functionals is itself a linear functional.

Each element $\gamma \in \bigwedge^{2} \mathfrak{g}^{*}$ can be viewed as a Poisson bivector. Indeed, $[\gamma, \gamma]=0$ since $\gamma$ is a constant tensor field. As such, $\gamma$ defines a Poisson structure on $\mathfrak{g}^{*}$. The condition of compatibility with the canonical LiePoisson structure, $\left[W_{0}, \gamma\right]=0$, can be written as (see [30], Chapter 3)

$$
\gamma([u, v], w)+\gamma([v, w], u)+\gamma([w, u], v)=0, \quad u, v, w \in \mathfrak{g} .
$$

On a Lie group $G$, a right-invariant $k$-form $\omega$ is completely defined by its value at the unit element $e$, and hence by an element of $\bigwedge^{k} \mathfrak{g}^{*}$. In other words, there is a natural isomorphism between the space of right-invariant $k$ forms on $G$ and $\bigwedge^{k} \mathfrak{g}^{*}$. Moreover, since the exterior differential $d$ commutes with right translations, it induces a linear operator $\partial: \bigwedge^{k} \mathfrak{g}^{*} \rightarrow \bigwedge^{k+1} \mathfrak{g}^{*}$ that satisfies $\partial \circ \partial=0$ and

(1) $\partial \gamma=0$ for $\gamma \in \bigwedge^{0} \mathfrak{g}^{*}=\mathbb{R}$;

(2) $\partial \gamma(u, v)=-\gamma([u, v])$ for $\gamma \in \bigwedge^{1} \mathfrak{g}^{*}=\mathfrak{g}^{*}$;

(3) $\partial \gamma(u, v, w)=\gamma([u, v], w)+\gamma([v, w], u)+\gamma([w, u], v)$ for $\gamma \in \bigwedge^{2} \mathfrak{g}^{*}$,

where $u, v, w \in \mathfrak{g}$, as one can check by direct computation (see [18], Chapter 24). The kernel $Z^{n}(\mathfrak{g})$ of $\partial: \bigwedge^{n}\left(\mathfrak{g}^{*}\right) \rightarrow \bigwedge^{n+1}\left(\mathfrak{g}^{*}\right)$ is the space of $n$ cocycles and the range $B^{n}(\mathfrak{g})$ of $\partial: \bigwedge^{n-1}\left(\mathfrak{g}^{*}\right) \rightarrow \bigwedge^{n}\left(\mathfrak{g}^{*}\right)$ is the spaces of $n$-coboundaries. Notice that $B^{n}(\mathfrak{g}) \subset Z^{n}(\mathfrak{g})$. The quotient space $H_{C E}^{n}(\mathfrak{g})=$ $Z^{n}(\mathfrak{g}) / B^{n}(\mathfrak{g})$ is the $n$-th Lie algebra cohomology or Chevaley-Eilenberg cohomology group of $\mathfrak{g}$. Notice that in general the Lie algebra cohomology is different from the de Rham cohomology $H_{D R}^{n}$. For example, $H_{D R}^{1}(\mathbb{R})=\mathbb{R}$ but $H_{C E}^{1}(\mathbb{R})=0$.

Each 2-cocycle $\gamma$ defines a Poisson structure on $\mathfrak{g}^{*}$ compatible with the canonical one. Indeed (2.13) can be recast as $\partial \gamma=0$. Notice that the Hamiltonian vector field $X_{f}$ of a function $f \in C^{\infty}\left(\mathfrak{g}^{*}\right)$ computed with respect to the Poisson structure defined by the 2-cocycle $\gamma$ is

$$
X_{f}(m)=\gamma\left(d_{m} f, \cdot\right) .
$$

Definition 2.9. A modified Lie-Poisson structure is a Poisson structure on $\mathfrak{g}^{*}$ whose Poisson bivector is given by $W_{\gamma}=W_{0}+\gamma$, where $W_{0}$ is the canonical Poisson bivector and $\gamma$ is a 2-cocycle.

\footnotetext{
${ }^{1}$ Here, $d_{m} f$, the differential of a function $f \in C^{\infty}\left(\mathfrak{g}^{*}\right)$ at $m \in \mathfrak{g}^{*}$ is to be understood as an element of the Lie algebra $\mathfrak{g}$
} 
Example 2.10. A special case of modified Lie-Poisson structure is given by a 2-cocycle $\gamma$ which is a coboundary. If $\gamma=\partial m_{0}$ for some $m_{0} \in \mathfrak{g}^{*}$, the expression

$$
\{f, g\}_{\gamma}(m)=m_{0}\left(\left[d_{m} f, d_{m} g\right]\right)
$$

looks like if the Lie-Poisson bracket had been "frozen" at a point $m_{0} \in \mathfrak{g}^{*}$ and for this reason some authors call it a "freezing" structure.

\section{Modified Lie-Poisson structures on $\operatorname{Vect}\left(\mathbb{S}^{1}\right)$}

The group Diff $\left(\mathbb{S}^{1}\right)$ of smooth orientation-preserving diffeomorphisms of the circle $\mathbb{S}^{1}$ is endowed with a smooth manifold structure based on the Fréchet space $\mathrm{C}^{\infty}\left(\mathbb{S}^{1}\right)$. The composition and the inverse are both smooth maps $\operatorname{Diff}\left(\mathbb{S}^{1}\right) \times \operatorname{Diff}\left(\mathbb{S}^{1}\right) \rightarrow \operatorname{Diff}\left(\mathbb{S}^{1}\right)$, respectively $\operatorname{Diff}\left(\mathbb{S}^{1}\right) \rightarrow \operatorname{Diff}\left(\mathbb{S}^{1}\right)$, so that Diff $\left(\mathbb{S}^{1}\right)$ is a Lie group [19]. Its Lie algebra $\operatorname{Vect}\left(\mathbb{S}^{1}\right)$ is the space of smooth vector fields on $\mathbb{S}^{1}$, which is isomorphic to the space $\mathrm{C}^{\infty}\left(\mathbb{S}^{1}\right)$ of periodic functions. The Lie bracket on $\operatorname{Vect}\left(\mathbb{S}^{1}\right)$ is given by

$$
[u, v]=u v_{x}-u_{x} v \text {. }
$$

Since the topological dual of the Fréchet space $\operatorname{Vect}\left(\mathbb{S}^{1}\right)$ is too big, being isomorphic to the space of distributions on the circle, we restrict our attention in the following to the regular dual Vect* $\left(\mathbb{S}^{1}\right)$, the subspace of distributions defined by linear functionals of the form

$$
u \mapsto \int_{\mathbb{S}^{1}} m u d x
$$

for some function $m \in \mathrm{C}^{\infty}\left(\mathbb{S}^{1}\right)$. The regular dual Vect* $\left(\mathbb{S}^{1}\right)$ is therefore isomorphic to $\mathrm{C}^{\infty}\left(\mathbb{S}^{1}\right)$ by means of the $L^{2}$ inner product ${ }^{2}$

$$
<u, v>=\int_{\mathbb{S}^{1}} u v d x .
$$

Let $f$ be a smooth real valued function on $\mathrm{C}^{\infty}\left(\mathbb{S}^{1}\right)$. Its Fréchet derivative at $m, d f(m)$ is a linear functional on $\mathrm{C}^{\infty}\left(\mathbb{S}^{1}\right)$. We say that $f$ is a regular function if there exists a smooth map $\delta f: \mathrm{C}^{\infty}\left(\mathbb{S}^{1}\right) \rightarrow \mathrm{C}^{\infty}\left(\mathbb{S}^{1}\right)$ such that

$$
d f(m) M=\int_{\mathbb{S}^{1}} M \cdot \delta f(m) d x, \quad m, M \in \mathrm{C}^{\infty}\left(\mathbb{S}^{1}\right) .
$$

That is, the Fréchet derivative $d f(m)$ belongs to the regular dual Vect* $\left(\mathbb{S}^{1}\right)$ and the mapping $m \mapsto \delta f(m)$ is smooth. The map $\delta f$ is a vector field on $\mathrm{C}^{\infty}\left(\mathbb{S}^{1}\right)$, called the gradient of $f$ for the $L^{2}$-metric. In other words, a regular function is a smooth function on $\mathrm{C}^{\infty}\left(\mathbb{S}^{1}\right)$ which has a smooth gradient.

Example 3.1. Typical examples of regular functions are nonlinear functionals over the space $\mathrm{C}^{\infty}\left(\mathbb{S}^{1}\right)$, like

$$
f(m)=\int_{\mathbb{S}^{1}}\left(m^{2}+m m_{x}^{2}\right) d x \quad \text { with } \quad \delta f(m)=2 m-m_{x}^{2}-2 m m_{x x},
$$

as well as linear functionals

$$
f(m)=\int_{\mathbb{S}^{1}} u m d x \quad \text { with } \quad \delta f(m)=u \in \mathrm{C}^{\infty}\left(\mathbb{S}^{1}\right) .
$$

\footnotetext{
${ }^{2}$ In the sequel, we use the notation $u, v, \ldots$ for elements of $\operatorname{Vect}\left(\mathbb{S}^{1}\right)$ and $m, n, \ldots$ for elements of Vect* $\left(\mathbb{S}^{1}\right)$ to distinguish them, although they all belong to $\mathrm{C}^{\infty}\left(\mathbb{S}^{1}\right)$.
} 
Notice that the smooth function $f_{\theta}: \mathrm{C}^{\infty}\left(\mathbb{S}^{1}\right) \rightarrow \mathbb{R}$ defined by $f_{\theta}(m)=m(\theta)$ for some fixed $\theta \in \mathbb{S}^{1}$ is not regular as $\delta f_{\theta}$ is the Dirac measure at $\theta$.

Conversely, a smooth vector field $X$ on $\operatorname{Vect}^{*}\left(\mathbb{S}^{1}\right)$ is called a gradient if there exists a regular function $f$ on Vect* $\left(\mathbb{S}^{1}\right)$ such that $X(m)=\delta f(m)$ for all $m \in \operatorname{Vect}^{*}\left(\mathbb{S}^{1}\right)$. Observe that if $f$ is a smooth real valued function on $\mathrm{C}^{\infty}\left(\mathbb{S}^{1}\right)$ then its second Fréchet derivative is symmetric [19], that is,

$$
d^{2} f(m)(M, N)=d^{2} f(m)(N, M), \quad m, M, N \in \mathrm{C}^{\infty}\left(\mathbb{S}^{1}\right) .
$$

For a regular function, this property can be written as

$$
\int_{\mathbb{S}^{1}}(d \delta f(m) M) N d x=\int_{\mathbb{S}^{1}}(d \delta f(m) N) M d x,
$$

for all $m, M, N \in \mathrm{C}^{\infty}\left(\mathbb{S}^{1}\right)$. Hence the linear operator $d \delta f(m)$ is symmetric for the $L^{2}$-inner product on $\mathrm{C}^{\infty}\left(\mathbb{S}^{1}\right)$ for each $m \in \mathrm{C}^{\infty}\left(\mathbb{S}^{1}\right)$. We will resume this fact in the following lemma.

Lemma 3.2. A necessary condition for a vector field $X$ on $\mathrm{C}^{\infty}\left(\mathbb{S}^{1}\right)$ to be a gradient is that its Fréchet derivative $d X(m)$ is a symmetric linear operator.

To define a Poisson bracket on the space of regular functions on Vect* $\left(\mathbb{S}^{1}\right)$, we consider a one-parameter family of linear operators $J(m)$ and set

$$
\{f, g\}(m)=\int_{\mathbb{S}^{1}} \delta f(m) J(m) \delta g(m) d x .
$$

The operators $J(m)$ must satisfy certain conditions in order for (3.2) to be a valid Poisson structure on Vect* $\left(\mathbb{S}^{1}\right)$.

Definition 3.3. A family of linear operators $J(m)$ on Vect* $\left.{ }^{*} \mathbb{S}^{1}\right)$ defines a Poisson structure on $\operatorname{Vect}^{*}\left(\mathbb{S}^{1}\right)$ if (3.2) satisfies

(1) $\{f, g\}$ is regular if $f$ and $g$ are regular,

(2) $\{g, f\}=-\{f, g\}$,

(3) $\{\{f, g\}, h\}+\{\{g, h\}, f\}+\{\{h, f\}, g\}=0$.

Notice that the second condition above simply means that $J(m)$ is a skew-symmetric operator for each $m$.

Example 3.4. The canonical Lie-Poisson structure on Vect* $\left(\mathbb{S}^{1}\right)$ given by

$$
\{f, g\}(m)=m([\delta f, \delta g])=\int_{\mathbb{S}^{1}} \delta f(m)(m D+D m) \delta g(m) d x
$$

is represented by the one-parameter family of skew-symmetric operators

$$
J(m)=m D+D m
$$

where $D=\partial_{x}$. It can be checked that all the three required properties are satisfied. In particular, we have

$$
\delta\{f, g\}=d \delta f(J \delta g)-d \delta g(J \delta f)+\delta f \delta g_{x}-\delta g \delta f_{x} .
$$

Definition 3.5. The Hamiltonian of a regular function $f$, for a Poisson structure defined by $J$ is defined as the vector field

$$
X_{f}(m)=J(m) \delta f(m) .
$$


Proposition 3.6. A necessary condition for a smooth vector field $X$ on Vect* $\left(\mathbb{S}^{1}\right)$ to be Hamiltonian with respect to the Poisson structure defined by a constant linear operator $K$ is the symmetry of the operator $d X(m) \circ K$ for each $m \in \operatorname{Vect}^{*}\left(\mathbb{S}^{1}\right)$.

Proof. If $X$ is Hamiltonian, we can find a regular function $f$ such that

$$
X(m)=K \delta f(m) .
$$

Moreover, since $K$ is a constant linear operator, we have

$$
d(K \delta f)(m) M=K \circ(d \delta f(m)) M .
$$

Therefore, we get

$$
\begin{aligned}
<d X(m) \circ K M, N> & =<K \circ d \delta f(m) \circ K M, N> \\
& =<M, K \circ d \delta f(m) \circ K N> \\
& =<M, d X(m) \circ K N>,
\end{aligned}
$$

since $K$ is skew-symmetric and $d \delta f(m)$ is symmetric.

A 2-cocycle on $\operatorname{Vect}\left(\mathbb{S}^{1}\right)$ is a bilinear functional $\gamma$ represented by a skewsymmetric operator $K: \mathrm{C}^{\infty}\left(\mathbb{S}^{1}\right) \rightarrow \mathrm{C}^{\infty}\left(\mathbb{S}^{1}\right)$ such that

$$
\gamma(u, v)=<u, K v>=\int_{\mathbb{S}^{1}} u K v d x,
$$

and satisfying the Jacobi identity

$$
<[u, v], K w>+<[v, w], K u>+<[w, u], K v>=0 .
$$

If $K$ is a differential operator we call $\gamma$ a differential cocycle. Gelfand and Fuks [16] observed that all differential 2-cocycles of $\operatorname{Vect}\left(\mathbb{S}^{1}\right)$ belong to the one-dimensional cohomology class generated by $\left[D^{3}\right]$. Moreover, each regular 2 -coboundary is represented by the skew-symmetric operator

$$
m_{0} D+D m_{0},
$$

for some $m_{0} \in \mathrm{C}^{\infty}\left(\mathbb{S}^{1}\right)$. Therefore, each differential 2-cocycle of $\operatorname{Vect}\left(\mathbb{S}^{1}\right)$ is represented by an operator of the form

$$
K=m_{0} D+D m_{0}+\beta D^{3}
$$

where $m_{0} \in \mathrm{C}^{\infty}\left(\mathbb{S}^{1}\right)$ and $\beta \in \mathbb{R}$ (see also [17]).

For $k \geq 0$ and $u, v \in \operatorname{Vect}\left(\mathbb{S}^{1}\right) \equiv \mathrm{C}^{\infty}\left(\mathbb{S}^{1}\right)$, let us now define the $H^{k}$ (Sobolev) inner product by

$$
<u, v>_{k}=\int_{\mathbb{S}^{1}} \sum_{i=0}^{k}\left(\partial_{x}^{i} u\right)\left(\partial_{x}^{i} v\right) d x=\int_{\mathbb{S}^{1}} A_{k}(u) v d x,
$$

where

$$
A_{k}=1-\frac{d^{2}}{d x^{2}}+\ldots+(-1)^{k} \frac{d^{2 k}}{d x^{2 k}}
$$

is a continuous linear isomorphism of $\mathrm{C}^{\infty}\left(\mathbb{S}^{1}\right)$. Note that $A_{k}$ is a symmetric operator for the $L^{2}$ inner product since

$$
\int_{\mathbb{S}^{1}} A_{k}(u) v d x=\int_{\mathbb{S}^{1}} u A_{k}(v) d x .
$$


The operator $A_{k}$ gives rise to a Hamiltonian function on $\operatorname{Vect}^{*}\left(\mathbb{S}^{1}\right)$ given by

$$
h_{k}(m)=\int_{\mathbb{S}^{1}} \frac{1}{2} m\left(A_{k}^{-1} m\right) d x
$$

The corresponding Hamiltonian vector field $X_{k}$ is given by

$$
X_{k}(m)=(m D+D m)\left(A_{k}^{-1} m\right)=2 m u_{x}+u m_{x},
$$

if we let $m=A_{k} u$.

Theorem 3.7. The Hamiltonian vector field $X_{k}$ is bi-Hamiltonian relatively to a modified Lie-Poisson structure if and only if $k \in\{0,1\}$.

Proof. It is well known (see [28]) that $X_{0}$ is bi-Hamiltonian with respect to the operator $D$ which represents a coboundary. It is also known that $X_{1}$ is a bi-Hamiltonian vector field with respect to the cocycle represented by the operator $D\left(1-D^{2}\right)$ cf. $[2,11,14]$. Notice that this cocycle is not a coboundary.

We will now show that there is no differential cocycle

$$
K=m_{0} D+D m_{0}+\beta D^{3}
$$

for which $X_{k}$ could be Hamiltonian unless $k \in\{0,1\}$. We have

$$
d X_{k}(m)=2 u_{x} I+u D+2 m D A_{k}^{-1}+m_{x} A_{k}^{-1},
$$

and in particular, for $m=1$,

$$
d X_{k}(1)=D+2 D A_{k}^{-1} \text {. }
$$

Letting

we obtain that

$$
P(m)=d X_{k}(m) \circ K
$$

$$
P(1)=\left(D+2 D A_{k}^{-1}\right) \circ\left(m_{0} D+D m_{0}\right)+\beta D^{4}\left(1+2 A_{k}^{-1}\right),
$$

whereas

$$
P(1)^{*}=\left(m_{0} D+D m_{0}\right) \circ\left(D+2 D A_{k}^{-1}\right)+\beta D^{4}\left(1+2 A_{k}^{-1}\right) .
$$

Therefore, denoting $m_{0}^{\prime}=\partial_{x} m_{0}$, we have

$$
\begin{aligned}
P(1)-P(1)^{*}=\left(m_{0}^{\prime} D+D m_{0}^{\prime}\right)+2\left(A_{k}^{-1} D m_{0} D-D m_{0} D A_{k}^{-1}\right)+ & \\
& +2\left(A_{k}^{-1} D^{2} m_{0}-m_{0} D^{2} A_{k}^{-1}\right) .
\end{aligned}
$$

If this operator is zero, we must have in particular the relation

$$
A_{k}\left(P(1)-P(1)^{*}\right) A_{k}\left(e^{i r x}\right)=0,
$$

for all $r \in \mathbb{Z}$. But, for $r \neq \pm 1$,

$$
A_{k}\left(e^{i r x}\right)=f_{k}(r) e^{i r x} \quad \text { with } \quad f_{k}(r)=\frac{r^{2 k+2}-1}{r^{2}-1},
$$

and

$$
A_{k}\left(P(1)-P(1)^{*}\right) A_{k}\left(e^{i r x}\right)
$$

is of the form $e^{i r x}$ times a polynomial expression in $r$ with highest order term $2 i m_{0}^{\prime}(x) r^{4 k+1}$. Therefore, a necessary condition for $X_{k}$ to be Hamiltonian relatively to the Poisson operator $K$ defined by (3.4) is that $m_{0}$ is a constant. 
Let $\alpha=2 m_{0} \in \mathbb{R}$. Then

$$
\begin{aligned}
P(m)=d X_{k}(m) \circ K=\alpha & \left\{2 u_{x} D+u D^{2}+2 m D^{2} A_{k}^{-1}+m_{x} D A_{k}^{-1}\right\}+ \\
& +\beta\left\{2 u_{x} D^{3}+u D^{4}+2 m D^{4} A_{k}^{-1}+m_{x} D^{3} A_{k}^{-1}\right\}
\end{aligned}
$$

because $D$ and $A_{k}$ commute. By virtue of Proposition 3.6, a necessary condition for $X_{k}$ to be Hamiltonian with respect to the cocycle represented by $K$ is that $P(m)$ is symmetric. That is

$$
<P(m) M, N>=<M, P(m) N>,
$$

for all $m, M, N \in \mathrm{C}^{\infty}\left(\mathbb{S}^{1}\right)$. Since this last expression is tri-linear in the variables $m, M, N$, the equality can be checked for complex periodic functions $m, M, N$ where the $L^{2}$ inner product is extended naturally into a complex bilinear functional. That is, the extension is not a hermitian product, we just allow homogeneity with respect to the complex scalar field in both components. Let $m=A_{k} u, u=\exp (i a x), M=\exp (i b x)$ and $N=\exp (i c x)$ with $a, b, c \in \mathbb{Z}$. We have

$$
\begin{aligned}
<P(m) M, N> & =\left[\left(2 a b^{3}+b^{4}\right) \beta-\left(2 a b+b^{2}\right) \alpha+\right. \\
& \left.+\left(\left(a b^{3}+2 b^{4}\right) \beta-\left(a b+2 b^{2}\right) \alpha\right) \frac{f_{k}(a)}{f_{k}(b)}\right] \int_{\mathbb{S}^{1}} e^{i(a+b+c) x} d x,
\end{aligned}
$$

whereas

$$
\begin{aligned}
<M, P(m) N> & =\left[\left(2 a c^{3}+c^{4}\right) \beta-\left(2 a c+c^{2}\right) \alpha+\right. \\
& \left.+\left(\left(a c^{3}+2 c^{4}\right) \beta-\left(a c+2 c^{2}\right) \alpha\right) \frac{f_{k}(a)}{f_{k}(c)}\right] \int_{\mathbb{S}^{1}} e^{i(a+b+c) x} d x .
\end{aligned}
$$

For $a=n, b=-2 n$ and $c=n$, we obtain

$$
<P(m) M, N>=\left(24 n^{4} \beta-6 n^{2} \alpha\right) \frac{f_{k}(n)}{f_{k}(2 n)},<M, P(m) N>=6 n^{4} \beta-6 n^{2} \alpha .
$$

The equality of the two expressions in (3.7) for all $n \in \mathbb{N}$ is ensured by means of (3.6). For $k=1$ this leads to the condition $\alpha+\beta=0$ and we recover the second Poisson structure given by $K=D-D^{3}$ for which $X_{1}$ is known to be Hamiltonian with Hamiltonian function

$$
\tilde{h}_{1}(m)=\frac{1}{2} \int_{\mathbb{S}^{1}}\left(\left(A_{1}^{-1} m\right)^{3}+\left(A_{1}^{-1} m\right)\left[\left(A_{1}^{-1} m\right)_{x}\right]^{2}\right) d x .
$$

In the general case, if $\beta \neq 0$, the leading term with respect to $n$ in the first expression in (3.7) is $\left(-48 \beta 2^{-2 k}\right)$, whereas in the second it is $(-12 \beta)$. Thus unless $\beta=0$ we must have $k=1$. On the other hand, if $\beta=0$, from (3.6)-(3.7) we infer that $\alpha f_{k}(n)=\alpha f_{k}(2 n)$ for all $n \in \mathbb{N}$. Thus $\alpha=0$ unless $k=0$. For $k=0$ we recover the Poisson structure given by $K=D$ for which $X_{0}$ is Hamiltonian with Hamiltonian function

$$
\tilde{h}_{0}(m)=\frac{1}{2} \int_{\mathbb{S}^{1}} m^{3} d x .
$$

This completes the proof. 


\section{Conclusion}

We showed that among all $H^{k}$ Sobolev inner products on $\mathrm{C}^{\infty}\left(\mathbb{S}^{1}\right)$, only for $k \in\{0,1\}$ is the associated vector field bi-Hamiltonian relatively to a modified Lie-Poisson structure. Endowing Diff $\left(\mathbb{S}^{1}\right)$ with the $H^{1}$ right-invariant metric, the associated geodesic equation turns out to be the Camassa-Holm equation [23] (see also [22])

$$
u_{t}+u u_{x}+\partial_{x}\left(1-\partial_{x}^{2}\right)^{-1}\left(u^{2}+\frac{1}{2} u_{x}^{2}\right)=0,
$$

a model for shallow water waves (see [2] and the alternative derivations in $[5,13,15,20])$ that accommodates waves that exist indefinitely in time $[3,7]$ as well as breaking waves $[6,8]$. The bi-Hamiltonian structure is reflected in the existence of infinitely many conserved integrals for the equation [2, $11,14,24]$ which are very useful in the qualitative analysis of its solutions. Both global existence results and blow-up results can be obtained using certain conservation laws $[3,7,31]$, while the proof of stability of traveling wave solutions relies on the specific form of some conserved quantities [4, $11,12,25]$. On the other hand, the geodesic equation on $\operatorname{Diff}\left(\mathbb{S}^{1}\right)$ for the $L^{2}$ right-invariant metric is the inviscid Burgers equation

$$
u_{t}+3 u u_{x}=0 .
$$

This model of gas dynamics has been thoroughly studied (see [9] and references therein). In contrast to the case of the $H^{1}$ right-invariant metric [10], the Riemannian exponential map is not a $C^{1}$ local diffeomorphism in the case of the $L^{2}$ right-invariant metric [9]. This means that of the two biHamiltonian vector fields $X_{0}$ and $X_{1}$, the second generates a flow on Diff $\left(\mathbb{S}^{1}\right)$ with properties that parallel those of geodesic flows on finite-dimensional Lie groups.

\section{REFERENCES}

[1] R. Abraham, J. E. Marsden, and T. S. Ratiu. Manifolds, tensor analysis, and applications. Springer-Verlag, New York, 1988.

[2] R. Camassa and D. D. Holm. An integrable shallow water equation with peaked solitons. Phys. Rev. Lett., 71:1661-1664, 1993.

[3] A. Constantin. On the Cauchy problem for the periodic Camassa-Holm equation. $J$. Differential Equations, 141:218-235, 1997.

[4] A. Constantin. On the inverse spectral problem for the Camassa-Holm equation. J. Funct. Anal., 155:352-363, 1998.

[5] A. Constantin. A Lagrangian approximation to the water-wave problem. Appl. Math. Lett., 14:789-795, 2001.

[6] A. Constantin and J. Escher. Wave breaking for nonlinear nonlocal shallow water equations. Acta Mathematica, 181:229-243, 1998.

[7] A. Constantin and J. Escher. Well-posedness, global existence, and blowup phenomena for a periodic quasi-linear hyperbolic equation. Comm. Pure Appl. Math., 51:475504, 1998.

[8] A. Constantin and J. Escher. On the blow-up rate and the blow-up set of breaking waves for a shallow water equation. Math. Z., 233:75-91, 2000.

[9] A. Constantin and B. Kolev. On the geometric approach to the motion of inertial mechanical systems. J. Phys. A, 35:R51-R79, 2002.

[10] A. Constantin and B. Kolev. Geodesic flow on the diffeomorphism group of the circle. Comment. Math. Helv., 78(4):787-804, 2003. 
[11] A. Constantin and H. P. McKean. A shallow water equation on the circle. Comm. Pure Appl. Math., 52:949-982, 1999.

[12] A. Constantin and W. A. Strauss. Stability of peakons. Comm. Pure Appl. Math., 53(5):603-610, 2000.

[13] A. S. Fokas. On a class of physically important integrable equations. Phys. D, 87:145150, 1995.

[14] A. S. Fokas and B. Fuchssteiner. Symplectic structures, their Bäcklund transformations and hereditary symmetries. Phys. D, 4:47-66, 1981/82.

[15] A. S. Fokas and Q. M. Liu. Asymptotic integrability of water waves. Phys. Rev. Lett., 77:2347-2351, 1996.

[16] I. M. Gelfand and D. B. Fuks. Cohomologies of the Lie algebra of vector fields on the circle. Funkcional. Anal. i Prilovzen., 2(4):92-93, 1968.

[17] L. Guieu and C. Roger. L'algèbre et le groupe de Virasoro. Les Publications CRM, Montreal, QC, 2007. Aspects géométriques et algébriques, généralisations. [Geometric and algebraic aspects, generalizations], With an appendix by Vlad Sergiescu.

[18] V. Guillemin and S. Sternberg. Symplectic techniques in physics. Cambridge University Press, Cambridge, 1990.

[19] R. S. Hamilton. The inverse function theorem of Nash and Moser. Bull. Amer. Math. Soc. (N.S.), 7:65-222, 1982.

[20] R. S. Johnson. Camassa-Holm, Korteweg-de Vries and related models for water waves. J. Fluid Mech., 455:63-82, 2002.

[21] R. Jost. Poisson brackets. Reviews Modern Physics, 36:572-579, 1964.

[22] B. Kolev. Lie groups and mechanics: an introduction. J. Nonlinear Math. Phys., 11:480-498, 2004.

[23] S. Kouranbaeva. The Camassa-Holm equation as a geodesic flow on the diffeomorphism group. J. Math. Phys., 40:857-868, 1999.

[24] J. Lenells. The correspondence between KdV and Camassa-Holm. Int. Math. Res. Not., (71):3797-3811, 2004.

[25] J. Lenells. Stability of periodic peakons. Int. Math. Res. Not., (10):485-499, 2004.

[26] A. Lichnerowicz. Les variétés de Poisson et leurs algèbres de Lie associées. J. Differential Geometry, 12(2):253-300, 1977.

[27] J. Milnor. Remarks on infinite-dimensional Lie groups. In Relativity, Groups and Topology, pages 1009-1057, Amsterdam, 1984. North-Holland.

[28] P. J. Morrison. Hamiltonian description of the ideal fluid. Rev. Modern Phys., 70:467$521,1998$.

[29] R. Schmid. Infinite dimensional Hamiltonian systems. Bibliopolis, Napoli, 1987.

[30] I. Vaisman. Lectures on the geometry of Poisson manifolds. Birkhäuser Verlag, Basel, 1994.

[31] E. Wahlén. A blow-up result for the periodic Camassa-Holm equation. Arch. Math. (Basel), 84:334-340, 2005.

Trinity College, Department of Mathematics, Dublin 2, Ireland

E-mail address: adrian@maths.tcd.ie

CMi, 39 rue F. Joliot-Curie, 13453 Marseille Cedex 13, France

E-mail address: boris.kolev@up.univ-mrs.fr 